Michelle Biehl, MD

Departments of Critical Care Medicine and Pulmonary

Medicine, Respiratory Institute, Cleveland Clinic
Denise Sese, MD

Departments of Critical Care Medicine and Pulmonary

Medicine, Respiratory Institute, Cleveland Clinic

\title{
Post-intensive care syndrome and COVID-19 - Implications post pandemic
}

\section{Posted July 22, 2020}

\section{ABSTRACT}

Post-intensive care syndrome (PICS) describes new or worsening physical, cognitive, or mental impairments in a patient following critical illness or intensive care. The COVID-19 pandemic will likely result in many more patients with PICS and its associated health and economic challenges. Screening and assessment tools should be utilized during hospitalization, at discharge, and post discharge to facilitate services and strategies to improve PICS outcomes for patients and their families.

\section{BACKGROUND}

Since the emergence of severe acute respiratory syndrome coronavirus (SARS-CoV) in 2003 and the 21st century's first pandemic, the $2009 \mathrm{H} 1 \mathrm{~N} 1$ influenza, it has become clear that the effects of serious, life threatening illness extend beyond the hospital stay and can have long-term sequelae for patients.

\section{PICS}

Post-intensive care syndrome (PICS) refers to a patient with new or worsening impairment in any physical, cognitive, or mental domain after critical illness or intensive care. These impairments persist beyond the intensive care unit (ICU) hospitalization $^{1,2}$ for as long as 5 to 15 years. ${ }^{3}$ The major risk factors for the development of PICS are acute respiratory distress syndrome (ARDS), sepsis, delirium, prolonged mechanical ventilation, and multiorgan failure. $^{3}$

Physical impairment in patients due to PICS is prevalent (up to $80 \%$ ) and includes muscular weak-

The statements and opinions expressed in COVID-19 Curbside Consults are based on experience and the available literature as of the date posted. While we try to regularly update this content, any offered recommendations cannot be substituted for the clinical judgment of clinicians caring for individual patients.

doi:10.3949/сcjm.87a.ccc055 ness, fatigue, dyspnea, impaired pulmonary function, decreased exercise tolerance, sexual dysfunction, and respiratory failure that frequently lead to a reduction in activities of daily living and quality of life.

Mental health impairment, including anxiety, depression and posttraumatic stress disorder (PTSD), occurs in $8 \%$ to $57 \%$ of patients with PICS and may also affect the patients' caregivers. Studies have noted a higher prevalence of post-ICU psychological sequelae in patients who are younger, female, have poor recall of the ICU stay, and had a longer duration of ICU sedation.

Finally, PICS cognitive impairment is noted in 30\% to $80 \%$ of patients and includes memory loss and difficulty with concentration, comprehension, and critical thinking. ${ }^{4}$ This is reflected in a study of over 800 patients with respiratory failure or shock admitted to intensive care units. ${ }^{5}$ Scores on global cognition were 1.5 standard deviation lower than the population and similar to patients with traumatic brain injury, with the duration of delirium independently associated with worse global cognition at 3 months and 1 year.

PICS impairments often last more than a year and have a profound impact on patients' quality of life, as well as that of their family members, known as PICS-F. Individuals with PICS-F are most commonly affected in the domain of mental health. As many as $40 \%$ of patients with PICS are unable to return to their former level of function, resulting in job loss and financial difficulties that can further complicate access to healthcare. Iatrogenic complications from polypharmacy and fragmentation of care also impact patient recovery as there is often a mismatch between the support needed relative to the support provided. ${ }^{6}$

The unrecognized needs of patients with PICS leads this vulnerable population to increased risk of subsequent hospitalization and emergency department and outpatient visits resulting in extraordinary healthcare-related costs. ${ }^{7}$ ARDS survivors are estimated to have a mean post-hospitalization cost of US $\$ 43,200$ per person. ${ }^{8}$ The cost burden of COVID19 survivors is expected to be higher. 


\section{PICS AND COVID-19}

Patients with severe illness due to COVID-19 often develop critical illness with hypoxemic respiratory failure, most commonly ARDS. ${ }^{9}$ Patients with COVID-19 treated in the ICU who survive may be at higher risk for developing PICS given the constraints on social support (restricted visitation), prolonged mechanical ventilation with exposure to higher amount of sedatives, and limited physical therapy during and after hospitalization given the risk of disease transmission. Post-ICU care for patients in rehabilitation centers, skilled nursing facilities, and long-term acute care hospitals is also subject to imposed service limitations due to exposure restrictions, limited personal protective equipment, and risk of transmission to caregivers. Furthermore, limited visitation policies due to the risk of transmission may increase the risk of PICS-F.

The scale of the pandemic foreshadows an incredible burden of patients with PICS with over 3.6 million cases and greater than 140,000 deaths in the United States due to COVID-19. In a study from the post-SARS era, it was observed that patients develop long-term fatigue, diffuse myalgia, weakness, depression, and sleep-disordered breathing. ${ }^{10} \mathrm{We}$ anticipate that not only will rehabilitation needs increase, but there will likely be higher rates of PTSD, depression, and substance abuse for patients, families, and health care workers, which is something that has been seen in large scale disasters such as $9 / 11 .{ }^{11}$

\section{During hospitalization and at time of discharge}

It is crucial that patients with critical illness or in intensive care are evaluated for the extent of physical, emotional, and cognitive impairments with ongoing assessment of patients' need for physical and occupational therapy, including signs of anxiety, depression, PTSD or cognitive difficulties. It is vital to utilize the daily ICU checklist and the ABCDEF bundle (daily assessment of pain, analgesia, sedation, liberation from mechanical ventilation, delirium, mobility, and family engagement) ${ }^{12}$ as well as assess nutrition and sleep.

Emerging data of patients recovering from COVID19 reveal 2 phases of the disease that have implications for rehabilitation. In the acute respiratory phase, early respiratory rehabilitation is highly recommended. The prolonged bedrest, immobilization phase warrants attention to neuromotor rehabilitation akin to acute stroke rehabilitation. ${ }^{13}$ This is especially true for older patients and those with obesity, multiple chronic diseases, or organ failure. With the prolonged recovery phase, a clinical field report noted greater than usual ICU(> 3 weeks) and hospital stays leading to greater debility, immobility, and complications in the midst of isolation. ${ }^{14}$ Rehabilitation and recovery was done at bedside with healthcare workers in personal protective equipment. ${ }^{13}$ There will likely be an increased need for mental health support as many will be grieving the loss of loved ones and experience unemployment, anxiety, and social isolation. ${ }^{15}$

\section{Post hospital discharge}

Patients with COVID-19 discharged from the hospital must be evaluated for the burden of care at home. COVID-19 has changed the healthcare landscape and may impact access to care for the elderly and minorities due to lost savings, unemployment, and inability to use technology for virtual outpatient visits. ${ }^{16}$

At the time of hospital follow-up, primary care providers (family medicine and internal medicine physicians) should be aware of and assess patients for signs and symptoms of PICS. Referral to an ICU recovery clinic could also help the physician manage the patient's complex and multidisciplinary physical and neuropsychiatric needs. The most recent literature shows that there are about 23 ICU recovery clinics in over 3 continents with more under development. ${ }^{17}$

At Cleveland Clinic, survivors of critical illness are offered an appointment at our post-ICU recovery clinic, which is an interdisciplinary clinic with a critical care physician, an advanced practice provider, a pharmacist, a mental health professional, a physical therapist, a respiratory therapist, and a case manager. The course of the ICU stay is discussed with the patient and family. Patients are evaluated and screened for mental health, cognitive, and physical impairments by optaining a history and physical exam, as well as use of screening tools such as the European Quality-of-Life Five Domains for mobility, self-care, usual activities, pain/discomfort, and anxiety/depression; the Hospital Anxiety and Depression Scale; the Impact of Events Scale-Revised for PTSD; and the Montreal Cognitive Assessment for cognitive impairment. ${ }^{18}$

Patients with COVID-19 who were critically ill and admitted to the ICU receive follow-up at the post-ICU recovery clinic 4 weeks after hospital discharge through virtual visits and in person visits. Patients often have many needs (Table 1) and including family members at follow-up visits allows providers to screen caregivers for distress, improve treatment adherence, and address gaps in social support. ${ }^{19}$ 
TABLE 1

\section{Patients needs revealed at post-ICU recovery clinic} visits

- Physical and occupational therapy

- Mental health support with referral to counseling, psychology, or psychiatry

- $\quad$ Detailed cognitive evaluation with referral to comprehensive neurocognitive testing as needed

- Medication review and reconciliation, initiation, or discontinuation

- Education of respiratory treatments such as inhalers and nebulizers

- $\quad$ Prescriptions for medical durable equipment

- Coordination of home health services for patients with physical impairments

- Referrals to specialists

- Coordination of care between providers

Thrive is an initiative of the Society of Critical Care Medicine created to facilitate strategies to improve outcomes of ICU survivors and their families. ${ }^{17,20}$ There is a Thrive learning collaborative for post-ICU care (https://www.sccm.org/MylCUCare/ Home) and a peer support collaborative (https:// www.facebook.com/ThriveICU/).

\section{REFERENCES}

1. Needham DM, Davidson J, Cohen H, et al. Improving long-term outcomes after discharge from intensive care unit: report from a stakeholders' conference. Crit Care Med. 2012;40(2):502-509. doi:10.1097/CCM.0b013e318232da75

2. Ohtake PJ, Coffey Scott J, Hinman RS, Lee AC, Smith JM. Impairments, activity limitations and participation restrictions experienced in the first year following a critical illness: protocol for a systematic review. BMJ Open. 2017;7(1):e013847. Published 2017 Jan 24. doi:10.1136/bmjopen-2016-013847

3. Desai SV, Law TJ, Needham DM. Long-term complications of critical care. Crit Care Med. 2011;39(2):371-379. doi:10.1097/ CCM.0b013e3181fd66e5

4. Colbenson GA, Johnson A, Wilson ME. Post-intensive care syndrome: impact, prevention, and management. Breathe (Sheff). 2019;15(2):98-101. doi:10.1183/20734735.0013-2019

5. Pandharipande PP, Girard TD, Jackson JC, et al. Long-term cognitive impairment after critical illness. N Engl J Med. 2013;369(14):13061316. doi:10.1056/NEJMoa1301372

6. Brown SM, Bose S, Banner-Goodspeed V, et al. Approaches to Addressing Post-Intensive Care Syndrome among Intensive Care Unit Survivors. A Narrative Review. Ann Am Thorac Soc. 2019;16(8):947-956. doi:10.1513/AnnalsATS.201812-913FR

7. Hill AD, Fowler RA, Pinto R, Herridge MS, Cuthbertson BH, Scales DC. Long-term outcomes and healthcare utilization following critical illness--a population-based study. Crit Care. 2016;20:76. Published 2016 Mar 31. doi:10.1186/s13054-016-1248-y
8. Gajic O, Ahmad SR, Wilson ME, Kaufman DA. Outcomes of critical illness: what is meaningful?. Curr Opin Crit Care. 2018;24(5):394400. doi:10.1097/MCC. 0000000000000530

9. Centers for Disease Control and Prevention. Interim Clinical Guidance for Management of Patients with Confirmed Coronavirus Disease (COVID-19). www.cdc.gov/coronavirus/2019-ncov/hcp/clinicalguidance-management-patients.html.Last Updated April 6; 2020. Accessed April 17, 2020.

10 Moldofsky H, Patcai J. Chronic widespread musculoskeletal pain, fatigue, depression and disordered sleep in chronic post-SARS syndrome; a case-controlled study. BMC Neurol. 2011;11:37. Published 2011 Mar 24. doi:10.1186/1471-2377-11-37

11 DePierro J, Lowe S, Katz C. Lessons learned from 9/11: Mental health perspectives on the COVID-19 pandemic. Psychiatry Res. 2020;288:113024. doi:10.1016/j.psychres.2020.113024

12 Marra A, Ely EW, Pandharipande PP, Patel MB. The ABCDEF Bundle in Critical Care. Crit Care Clin. 2017;33(2):225-243. doi:10.1016/j. ccc.2016.12.005

13 Brugliera L, Spina A, Castellazzi P, et al. Rehabilitation of COVID-19 patients. J Rehabil Med. 2020;52(4):jrm00046. Published 2020 Apr 15. doi:10.2340/16501977-2678

14 Kiekens C, Boldrini P, Andreoli A, et al. Rehabilitation and respiratory management in the acute and early post-acute phase. "Instant paper from the field" on rehabilitation answers to the Covid-19 emergency [published online ahead of print, $2020 \mathrm{Apr}$ 15]. Eur J Phys Rehabil Med. 2020;10.23736/S1973-9087.20.06305-4. doi:10.23736/S1973-9087.20.06305-4

15 Pompeo-Fargnoli A, Fargnoli AS. The Mental Health Impact of the COVID19 Crisis: The Battle Ahead for Inpatient Survivors [published online ahead of print, 2020 Apr 8]. Psychosomatics. 2020;500333182(20)30064-5. doi:10.1016/j.psym.2020.04.001

16 Morrow-Howell N, Galucia N, Swinford E. Recovering from the COVID-19 Pandemic: A Focus on Older Adults [published online ahead of print, 2020 Apr 26]. J Aging Soc Policy. 2020;1-9. doi:10.10 80/08959420.2020.1759758

17 Haines KJ, McPeake J, Hibbert E, et al. Enablers and Barriers to Implementing ICU Follow-Up Clinics and Peer Support Groups Following Critical Illness: The Thrive Collaboratives. Crit Care Med. 2019;47(9):1194-1200. doi:10.1097/CCM.0000000000003818

18 Needham DM, Sepulveda KA, Dinglas VD, et al. Core Outcome Measures for Clinical Research in Acute Respiratory Failure Survivors. An International Modified Delphi Consensus Study. Am J Respir Crit Care Med. 2017;196(9):1122-1130. doi:10.1164/ rccm.201702-03720C

19 Herridge MS, Moss M, Hough CL, et al. Recovery and outcomes after the acute respiratory distress syndrome (ARDS) in patients and their family caregivers. Intensive Care Med. 2016;42(5):725-738. doi:10.1007/s00134-016-4321-8

20 McPeake J, Hirshberg EL, Christie LM, et al. Models of Peer Support to Remediate Post-Intensive Care Syndrome: A Report Developed by the Society of Critical Care Medicine Thrive International Peer Support Collaborative. Crit Care Med. 2019;47(1):e21-e27. doi:10.1097/ CCM.0000000000003497 